\title{
Electron and hole states in diluted magnetic semiconductor quantum dots
}

\author{
Kai Chang,* S. S. Li, and J. B. Xia \\ NLSM, Institute of Semiconductors, Chinese Academy of Sciences, P.O. Box 912, Beijing 100083, China \\ F. M. Peeters \\ Department of Physics, University of Antwerp (Campus Drie Eiken), B-2610 Antwerpen, Belgium
}

(Received 15 December 2003; published 17 June 2004)

\begin{abstract}
The electronic structure of a diluted magnetic semiconductor (DMS) quantum dot (QD) is studied within the framework of the effective-mass theory. We find that the energies of the electron with different spin orientation exhibit different behavior as a function of magnetic field at small magnetic fields. The energies of the hole decreases rapidly at low magnetic fields and saturate at higher magnetic field due to the $s p-d$ exchange interaction between the carriers and the magnetic ions. The mixing effect of the hole states in the DMS QD can be tuned by changing the external magnetic field. An interesting crossing behavior of the hole ground state between the heavy-hole state and the light-hole state is found with variation of the QD radius. The strength of the interband optical transition for different circular polarization exhibts quite different behavior with increasing magnetic field and QD radius.
\end{abstract}

DOI: 10.1103/PhysRevB.69.235203

PACS number(s): 78.20.Ls, 78.67.Hc, 78.55.Cr

\section{INTRODUCTION}

The interest in the spin dynamics of carriers in semiconductor structures has increased remarkably because of its importance for basic physics as well as for its potential application in spintronic devices. Several proposals ${ }^{1,2}$ for quantum information storage and processing using (electron or nuclear) spins in semiconductor quantum dots (QD) have been put forward due to the long spin-coherence time in semiconductors. Quantum information processing should preserve the entanglement while the quantum information is transferred from the photon system to the spin of the electron in the semiconductor. This process is closely related to the spin splitting of carriers in semiconductors, i.e., the effective $g$ factor of the carrier or the exciton. Due to the strong $s p-d$ interaction between the carriers and the magnetic ions, diluted magnetic semiconductor (DMS) structures ${ }^{3,4}$ provides us with a unique flexibility to tailor the spin splitting of carriers in DMS systems via the external magnetic field. ${ }^{5}$ The external magnetic field induces a magnetization of the magnetic ions in the DMS which gives rise to a giant spin splitting of the electron and hole band structure via the exchange interaction. Very recently, incorporation of $\mathrm{Mn}$ ions into the crystal matrix of different II-VI semiconductors, successful approaches to fabricate DMS quantum dot and magnet/DMS hybrid structures has been reported. ${ }^{6-8}$ Photoluminescence (PL) signals clearly demonstrated the transition of quasizero-dimensional electron-hole pairs bound to these nanostuctures. Due to the requirement of a quantitative understanding of the optical properties of DMS QD, there arises a fundamental interest in the electronic structure of DMS quantum dots.

In the case of semiconductor nanostructures, the electronic structure varies significantly with decreasing size of the semiconductor nanostructures, especially for the hole states. In the zinc-blende bulk material, the heavy- and lighthole are degenerate with vanishing momentum since the Luttinger Hamiltonian describing the hole states becomes diag- onal with vanishing momentum. In the quantum well case, the heavy- and light-hole are nondegenerate due to the confinement along the growth direction. But the projection of the angular momentum of the band-edge Bloch state on the growth direction $J_{z}$ is still a constant of motion. In quantum dot structures, the situation is very different due to the threedimensional quantum confinement. $J_{z}$ is no longer a good quantum number due to the band mixing effect: the hole eigenstates become mixtures of the heavy- and light- hole states. In a DMS QD, an external magnetic field induces a magnetization of the magnetic ions, and the strong exchange interaction between carriers and the magnetic ions provides us with a unique and interesting flexibility to tailor the electronic structure of the DMS QD, consequently changing the optical property of the DMS QD, i.e., the polarization and energy position of the PL signals. In this paper, we investigate theoretically the electronic structure of a DMS $\mathrm{Cd}_{1-x} \mathrm{Mn}_{x} \mathrm{Te} / \mathrm{Cd}_{1-y} \mathrm{Mg}_{y} \mathrm{Te} \mathrm{QD}$. We show the energies of the lowest hole states as function of the magnetic field and the confinement. The energy of the hole decreases rapidly at low magnetic fields and saturates at high magnetic field. An interesting crossing behavior between the heavy-hole and light-hole is found with variation of the in-plane confinement. The strength of the interband optical transition for different circular polarization exhibits quite different behavior with increasing magnetic field.

The paper is organized as follows: the model and formalism are presented in Sec. II, in Sec. III we show the numerical results along with the discussions. A brief conclusion is given in Sec. IV.

\section{MODEL AND FORMALISM}

The DMS quantum dot is constructed from a DMS $\mathrm{Cd}_{1-x} \mathrm{Mn}_{x} \mathrm{Te} / \mathrm{Cd}_{1-y} \mathrm{Mg}_{y} \mathrm{Te}$ quantum well with a lateral confinement of the carriers through a parabolic well where the $z$ axis will be taken along the growth direction. The electron Hamiltonian is 


$$
H_{e}=\frac{(\mathbf{p}+e \mathbf{A})^{2}}{2 m_{e}^{*}}+V_{e}(\rho, z)+J_{s-d} \mathbf{S} \cdot \sigma_{e} \pm g_{e}^{*} \mu_{B} B / 2,
$$

where $m_{e}^{*}$ is the effective mass of the electron in units of the free electron mass $m_{0}, \mathbf{A}=(-y, x, 0) B / 2$ is the vector potential in the symmetric gauge. $V_{e}=V_{\|}^{e}+V_{\perp}^{e}$ is the confining potential of the electron which will be given explicitly, the third term describes the exchange interaction between electron and the magnetic ions in the DMS QD, the last term of the above equation gives the intrinsic Zeeman splitting.

Within the axial approximation, the hole Hamiltonian in the DMS QD can be written as ${ }^{9-13}$

$$
H_{h}=\frac{\hbar^{2}}{2 m_{0}}\left(\begin{array}{cccc}
H_{h h} & R & S & 0 \\
R^{*} & H_{l h} & 0 & S \\
S^{*} & 0 & H_{l h} & -R \\
0 & S^{*} & -R^{*} & H_{h h}
\end{array}\right)+V_{h}(\rho, z)+J_{p-d} \mathbf{S} \cdot \sigma_{h},
$$

where

$$
\begin{gathered}
H_{h h}=\left(\gamma_{1}+\gamma_{2}\right)\left(k_{x}^{2}+k_{y}^{2}\right)+\left(\gamma_{1}-2 \gamma_{2}\right) k_{z}^{2}+E_{Z}, \\
H_{l h}=\left(\gamma_{1}-\gamma_{2}\right)\left(k_{x}^{2}+k_{y}^{2}\right)+\left(\gamma_{1}+2 \gamma_{2}\right) k_{z}^{2}+E_{Z}, \\
R=2 \sqrt{3} \gamma_{3} i k_{-} k_{z}, \\
S=\sqrt{3} \gamma k_{-}^{2} .
\end{gathered}
$$

Here $\mathbf{k}=-i \nabla-e \mathbf{A} / \hbar$, and $k_{ \pm}=k_{x} \pm i k_{y}, \gamma_{1}, \gamma_{2}$, and $\gamma_{3}$ are the Luttinger parameters, $\gamma=\left(\gamma_{2}+\gamma_{3}\right) / 2$. $E_{Z}=-\left(\hbar e / m_{0}\right) \kappa B j_{z}$ describes the Zeeman splitting of the hole, $\kappa$ is another Luttinger parameter, the confining potential $V_{h}=V_{\|}^{h}+V_{\perp}^{h}, V_{\|}^{e, h}$ is the lateral confining potential of electron or hole in the DMS QD,

$$
V_{\|}^{e, h}\left(\rho_{e, h}, z_{e, h}\right)=\frac{1}{2} m_{e, h} \omega_{e, h}^{2} \rho_{e, h}^{2},
$$

and a quantum well potential confinement is assumed in the $z$ direction,

$$
V_{\perp}^{e, h}\left(z_{e, h}\right)= \begin{cases}\Delta V^{e, h}, & |z| \geqslant w / 2, \\ 0, & |z|<w / 2,\end{cases}
$$

where $\rho_{i}$ and $z_{i}$ denote the cylinderical coordinates of the electron or hole. $w$ is the height of the DMS QD. $\Delta V^{i}$ is the band offset of electron or hole. Notice that the zero energy for the electron and hole are at the bottom of the conduction band and the top of the valence band of $\mathrm{Cd}_{1-x} \mathrm{Mn}_{x} \mathrm{Te}$, respectively. The exchange interaction term describes the $s p-d$ exchange interaction between the carriers and the magnetic ion $\mathrm{Mn}^{2+}$ and can be expressed as within the mean-field approximation,

$$
V_{\mathrm{exch}}^{e, h}=J_{s p-d}^{e, h}\left\langle S_{z}\right\rangle J_{z},
$$

where $\quad J_{s-d}=N_{0} \alpha x_{\text {eff }} / 2, \quad J_{p-d}=-N_{0} \beta x_{\text {eff }} / 3, \quad$ and $\left\langle S_{z}\right\rangle$ $=5 / 2 B_{J}\left(S g_{\mathrm{Mn}} \mu_{B} B / k_{B}\left(T+T_{0}\right)\right), S=5 / 2$ corresponds to the spins of the localized $3 d^{5}$ electrons of the $\mathrm{Mn}^{2+}$ ions. $B_{J}(x)$ is the Brillouin function, $N_{0}$ is the number of cations per unit volume, the phenomenological parameters $x_{\text {eff }}$ (reduced ef- fective concentration of $\mathrm{Mn}$ ) and $T_{0}$ accounts for the reduced single-ion contribution due to the antiferromagnetic $\mathrm{Mn}-\mathrm{Mn}$ coupling, $k_{B}$ is the Boltzmann constant, $\mu_{B}$ the Bohr magneton, $g_{\mathrm{Mn}}=2$ is the effective $g$ factor of $\mathrm{Mn}^{2+}$ ion and $J_{z}$ $= \pm 1 / 2, \pm 3 / 2$ is the hole spin. $B$ is the external magnetic field.

In the axial approximation the hole Hamiltonian is rotationally invariant around the $z$ axis, therefore the projection $f_{z}$ of the total angular momentum $\mathbf{F}=\mathbf{L}+\mathbf{J}$ on the $z$ axis is a good quantum number, where $\mathbf{J}$ is the angular momentum of the band-edge Bloch function and $\mathbf{L}$ is the envelope angular momentum. The single-particle eigenstates of the electron and hole with, respectively, the angular quantum number $l$ and $f_{z}$ are expanded in the basis of the two-dimensional harmonic oscillator function

$$
\psi_{l}^{e}=\sum_{n, s} c_{n l} \Psi_{n, l}(\rho, \phi) f_{s}(z)\left|1 / 2, \sigma_{z}\right\rangle
$$

and

$$
\psi_{f_{Z}}^{h}=\sum_{j_{z}, n, s} c_{n s j_{Z}} \Psi_{n, f_{Z}-j_{Z}}(\rho, \phi) f_{s}(z)\left|3 / 2, j_{z}\right\rangle
$$

where $f_{s}(z), s=1,2 \ldots$ is a convenient basis for the subband part,

$$
f_{s}(z)=\sqrt{\frac{2}{L}} \sin \left[\frac{s \pi}{L}\left(x+\frac{L}{2}\right)\right], \quad|z| \leqslant L / 2,
$$

and zero otherwise. $L$ is allowed to be larger than the height $w$ of the DMS QD. The number of terms in the summation $s$ is determined by the convergence of the subband energy. $\left|1 / 2, \sigma_{z}\right\rangle$ and $\left|3 / 2, j_{z}\right\rangle$ are the band-edge Bloch functions of electron and hole, the oscillator function $\Psi_{n, l}(\rho, \phi)$ is

$$
\Psi_{n, l}(\rho, \phi)=C_{n l}(i \rho)^{|l|} e^{-\rho^{2} / 2 a^{2}} e^{i l \phi} L_{n}^{|l|}\left(\rho^{2} / a^{2}\right),
$$

where $C_{n l}$ is the normalization constant and $L_{n}^{|l|}$ is the generalized Laguerre polynomial, $a_{e, h}$ is the length related to the magnetic length $a_{c}=(\hbar / e B)^{1 / 2}$ and the confinement length $l_{e, h}=\left(\hbar / m_{e, h}^{*} \omega_{0}\right)^{1 / 2}$ by $a_{e, h}=\sqrt{2} l_{e, h} a_{c} /\left(l_{e, h}^{4}+4 a_{c}^{4}\right)^{1 / 4}$.

The dipole optical transition probability for photoemission is proportional to the optical transition matrix element

$$
G=\frac{2}{m_{0}}\left|\epsilon \cdot\left\langle\psi_{l}^{e}|\mathbf{P}| \psi_{f_{Z}}^{h}\right\rangle\right|^{2},
$$

where $\epsilon$ is the unit vector along the direction of the electric field component and $\psi_{l}^{e}\left(\psi_{f_{Z}}^{h}\right)$ is the electron (hole) wave fucntion. When the light propagates in the direction of the magnetic field, i.e., $z$ axis, the $\sigma^{ \pm}$polarizations [electric field polarization $\left.\epsilon_{ \pm}=(1 / \sqrt{2})\left(\epsilon_{x} \pm i \varepsilon_{y}\right)\right]$ is of interest. Therefore the corresponding matrix elements between the spin-down electron state and the hole states with $J_{z}=1 / 2,-3 / 2$ components are

$$
G_{+}=\frac{2 P^{2}}{m_{0}}\left|\left\langle\psi_{-1 / 2}^{e} \mid \psi_{-3 / 2}^{h}\right\rangle\right|^{2},
$$




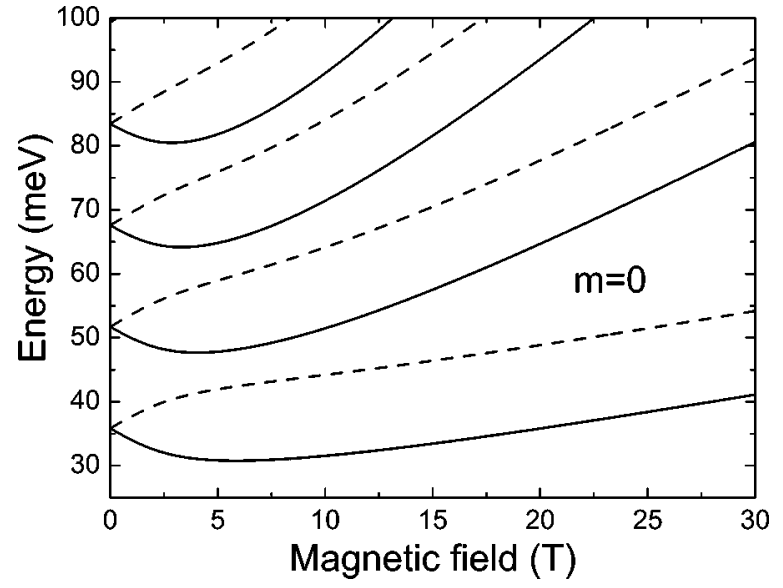

FIG. 1. The energy of the lowest eight electron states in a DMS QD versus magnetic field. The QD radius is $10 \mathrm{~nm}$ and the thickness of the QD is $10 \mathrm{~nm}$. The solid lines and the dashed lines denote the energies of the spin-down and the spin-up states, respectively.

$$
G_{-}=\frac{2 P^{2}}{3 m_{0}}\left|\left\langle\psi_{-1 / 2}^{e} \mid \psi_{1 / 2}^{h}\right\rangle\right|^{2},
$$

and the matrix elements between the spin-up electron and the hole states with $J_{z}=-1 / 2,3 / 2$ components are

$$
G_{+}=\frac{2 P^{2}}{m_{0}}\left|\left\langle\psi_{1 / 2}^{e} \mid \psi_{3 / 2}^{h}\right\rangle\right|^{2},
$$

$$
G_{-}=\frac{2 P^{2}}{3 m_{0}}\left|\left\langle\psi_{1 / 2}^{e} \mid \psi_{-1 / 2}^{h}\right\rangle\right|^{2},
$$

$P=\left\langle s\left|p_{x}\right| X\right\rangle, s$ and $X$ are the Bloch functions at the bottom of the conduction band and the top of the valence band, respectively.

The parameters used in our calculation are $m_{e}^{*}=0.13 m_{0}$, $\gamma_{1}=4.02, \gamma_{2}=1.37, \gamma_{3}=1.64, \kappa=0.617, x_{\mathrm{eff}}=0.045, g_{\mathrm{Mn}}=2$, $N_{0} \alpha=0.27 \mathrm{eV}, N_{0} \beta=-1.31 \mathrm{eV}$, and $T_{0}=3.6 \mathrm{~K}$.

\section{NUMERICAL RESULTS AND DISCUSSIONS}

In this section we present our numerical results on the electron and hole states in $\mathrm{Cd}_{1-x} \mathrm{Mn}_{x} \mathrm{Te} / \mathrm{Cd}_{1-y} \mathrm{Mg}_{y}$ Te DMS QDs. The magnetic field is applied perpendicular to the $x-y$ plane. The energies of the lowest eight electron and hole states in the DMS QD are plotted in Figs. 1 and 2(a)-2(d) as a function of magnetic field. From Fig. 1, it is apparent that at small magnetic field the energies of spin-down (spin-up) electron states decrease (increase) with increasing magnetic fields. But at high magnetic fields they all increase. At small magnetic field the energies are determined by the exchange interaction term [Eq. (5)] for spin-up and spin-down states. At large magnetic field the exchange term approaches to a constant and the energies are determined mainly by the magnetic confinement term. Due to the rotational symmetry around the $z$ axis, the projection of the total angular momentum $f_{z}$ is a constant of motion, the hole eigenstate $S_{f_{z}}$ is labeled by its total angular momentum $f_{z}$ and the dominant term in Eq. (6b) is usually the term with the smallest $|l|$. The behavior of the energies of the hole states [see Figs.

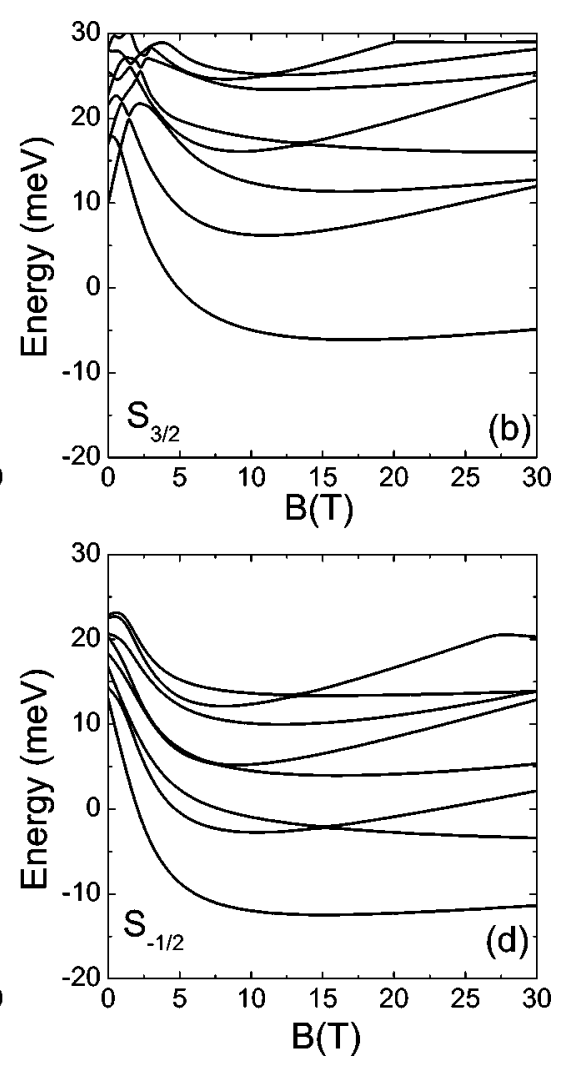

FIG. 2. The same as Fig. 1 but now for the different hole states $S_{f_{z}}$. 

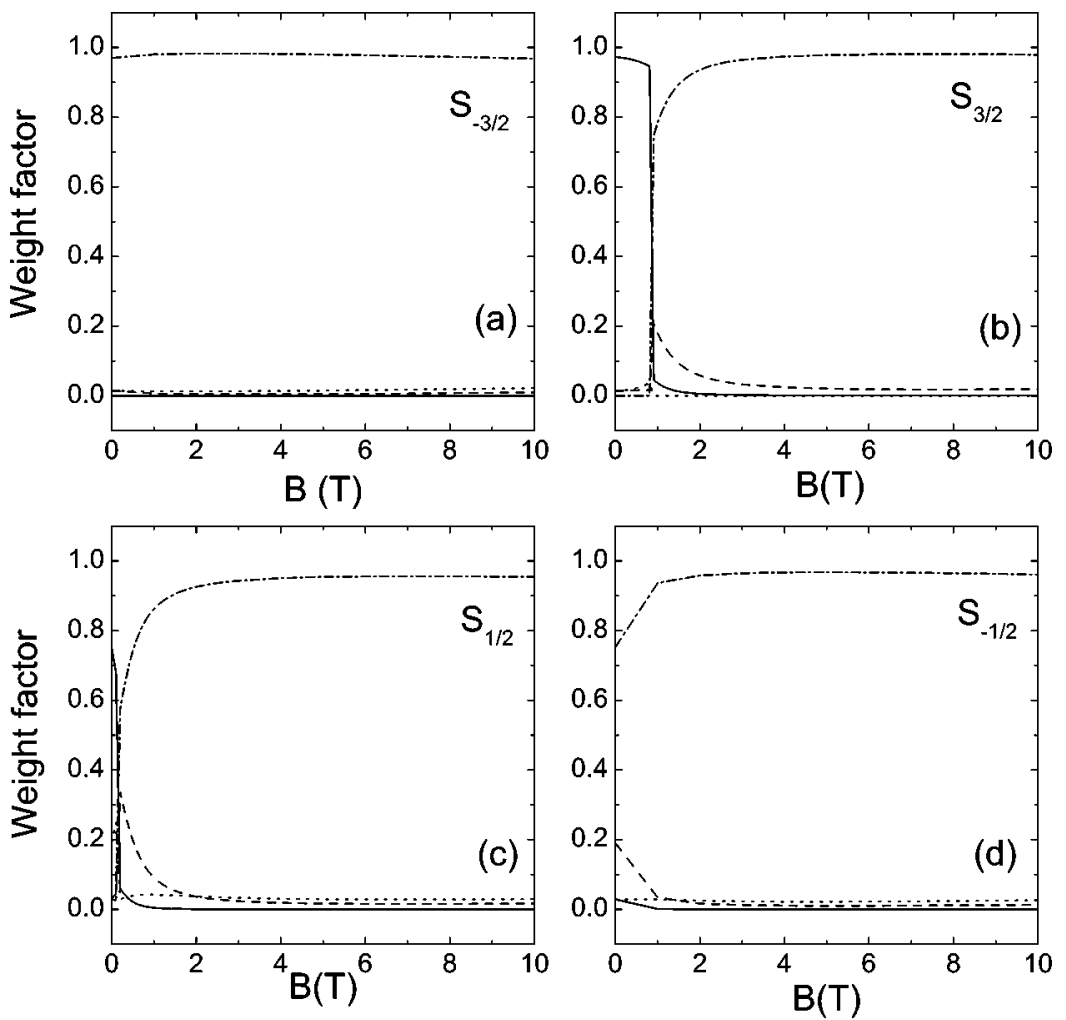

FIG. 3. The weight factor (a)-(d) and the expected value of the angular momentum $\left\langle J_{z}\right\rangle$ (e) of the hole ground state for different angular momentum $F$ in a DMS QD versus the magnetic field. The solid, dashed, dotted, and dasheddotted curves in (a)-(d) correspond to the hole components $|3 / 2,-3 / 2\rangle,|3 / 2,-1 / 2\rangle,|3 / 2,1 / 2\rangle$, $|3 / 2,3 / 2\rangle$, respectively. The QD structure is the same as in Fig. 1.

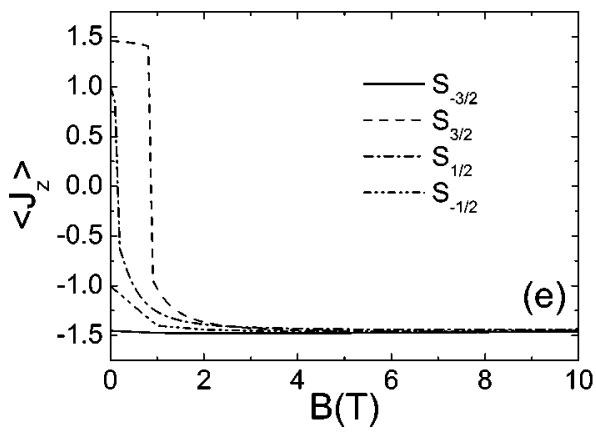

2(a)-2(d)] is much more complicated, they decrease rapidly with increasing magnetic field at small magnetic field, and saturate at high magnetic fields. The hole states are more complicated than the electron states, this is because the wave function of the hole state consists of four components, each component has a different $J_{z}$ and $l$, and they mix with each other due to the contribution of the off-diagonal terms in the hole Hamiltonian [Eq. (2)]. The exchange interaction term and the Zeeman term cause splitting of the energies of the four components at small magnetic field. At large magnetic field the hole energies increase slightly due to the magnetic confinement, but is different for different states $S_{f_{z}}$, i.e., different orbital momentum $l$. Since the spacing of the bound states is comparable with that of the magnetic energy level in the $x-y$ plane and the heavy- and light-hole mix with each other which is induced by the contribution of the offdiagonal terms in the hole Hamiltonian [Eq. (2)], therefore there appear many crossings and anticrossings between energy levels as shown in Figs. 3(c) and 3(d). Notice that the $S_{3 / 2}$ and $S_{-3 / 2}\left(S_{1 / 2}\right.$ and $\left.S_{-1 / 2}\right)$ states are degenerate at $B=0$ and the energy of the $S_{3 / 2}$ and $S_{1 / 2}$ ground state exhibits a local maximum at small magnetic fields. This arises from a crossover from the heavy-hole component $|3 / 2,3 / 2\rangle$ with $l$ $=0$ to $|3 / 2,-3 / 2\rangle$ with $l=3$, the latter becomes dominant with increasing magnetic fields due to the off-diagonal terms in the hole Hamiltonian (see Fig. 2). The slight increase of the energies of the lowest states with different angular momentum $\left(S_{3 / 2}, S_{-3 / 2}, S_{1 / 2}, S_{-1 / 2}\right)$ at high magnetic fields is caused by the magneto-confinement effect and the intrinsic Zeeman effect.

Figures 3(a)-3(e) show the weight factors of the wave functions of hole ground states with $f_{z}=-3 / 2,3 / 2,1 / 2$, $-1 / 2$, i.e., the relative contribution of the hole components with angular momentum $J_{z}$, and the average value of the angular momentum $\left\langle J_{z}\right\rangle$ in the DMS QD as a function of the magnetic field. Notice that the magnetic-field dependence of the weight factor of the hole components with different angular momentum $f_{z}$ exhibit very different behavior. For the $S_{-3 / 2}$ state, the weight factor of the heavy-hole component $|3 / 2,-3 / 2\rangle$ is dominant and stays almost constant with increasing magnetic field, but for the $S_{3 / 2}$ state, the dominant component is the heavy-hole component $|3 / 2,3 / 2\rangle$ at small 


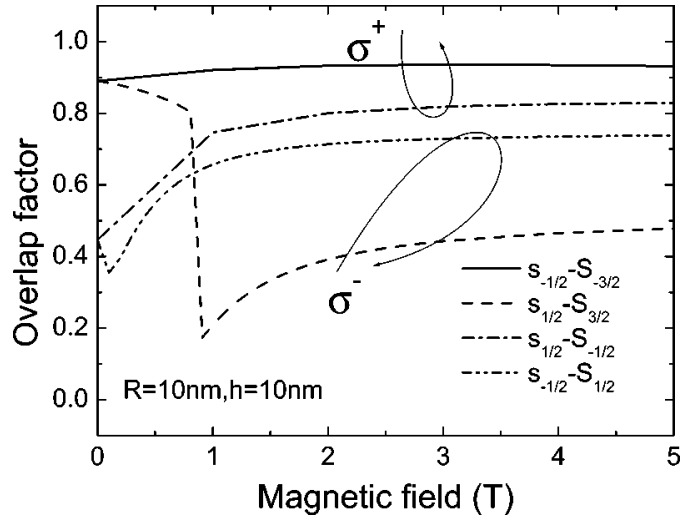

FIG. 4. The overlap factor of the electron and hole in the DMS QD versus the magnetic field. The QD structure is the same as in Fig. 1.

magnetic fields but change rapidly to the component $3 / 2$, $-3 / 2\rangle$ at higher magnetic fields. The main relative contributions of the $S_{1 / 2}$ and $S_{-1 / 2}$ states come from the components $|3 / 2,1 / 2\rangle$ and $|3 / 2,-3 / 2\rangle$, and the latter becomes dominant with increasing magnetic field. The expected value of the angular momentum $\left\langle J_{z}\right\rangle$ is plotted in Fig. 3(e) as a function of magnetic field. In QD structures, $J_{z}$ is no longer a good quantum number due to the band mixing effect that exists even at vanishing momentum. For these four hole ground states, $\left\langle J_{z}\right\rangle$ decreases with increasing magnetic fields and keep constant $-3 / 2$ at high magnetic fields. This phenomenon can be understood from the weight factors of the different hole components in the hole eigenstates [see Figs. 2(a)-2(d)]. Since the dominant component of the hole eigenstates at high magnetic field is the spin-down heavy holes $|3 / 2,-3 / 2\rangle$, therefore $\left\langle J_{z}\right\rangle$ of these four hole eigenstates is equal to $-3 / 2$. It is interesting to find that $\left\langle J_{z}\right\rangle$ of the hole eigenstate $S_{-3 / 2}$ experiences a sharp change from $3 / 2$ to

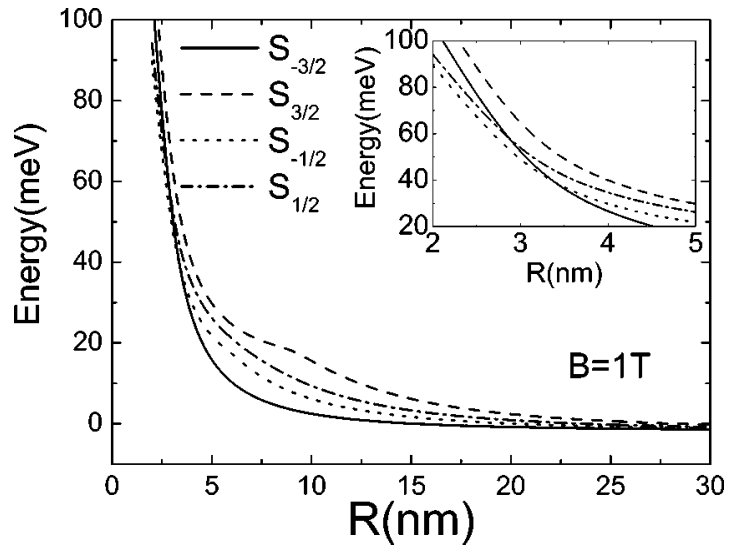

FIG. 6. The energies of the electron and the hole ground states in DMS QD versus QD radius. The thickness of the QD is $10 \mathrm{~nm}$ and $B=1 \mathrm{~T}$.

$-3 / 2$ with increasing magnetic field due to the crossover of the hole components $|3 / 2,3 / 2\rangle$ and $|3 / 2,-3 / 2\rangle$, and a similar behavior can also be found for the $S_{1 / 2}$ state.

In Fig. 4 we plot the overlap factor $\left|\left\langle\psi_{\sigma}^{e} \mid \psi_{f z}^{h}\right\rangle\right|$, i.e., the interband transition strength for different circular polarization of the light [see Eqs. (8) and (9)] as a function of magnetic field. The overlap factors for different transitions between the electron states and the hole states exhibit different behavior as a function of magnetic field. The biggest difference can be found at small magnetic fields. This difference is easily understood from the wave functions of the electron and hole eigenstates. The wave functions of hole ground states are shown in Figs. 5(a)-5(d) for different magnetic fields. The wave functions of the $S_{-3 / 2}$ and $S_{-1 / 2}$ states change slightly for different magnetic fields, but the wave functions of the $S_{3 / 2}$ and $S_{1 / 2}$ states varies significantly with changing magnetic field. Since the electron always localizes at the center of the DMS QD for different magnetic fields, and the hole
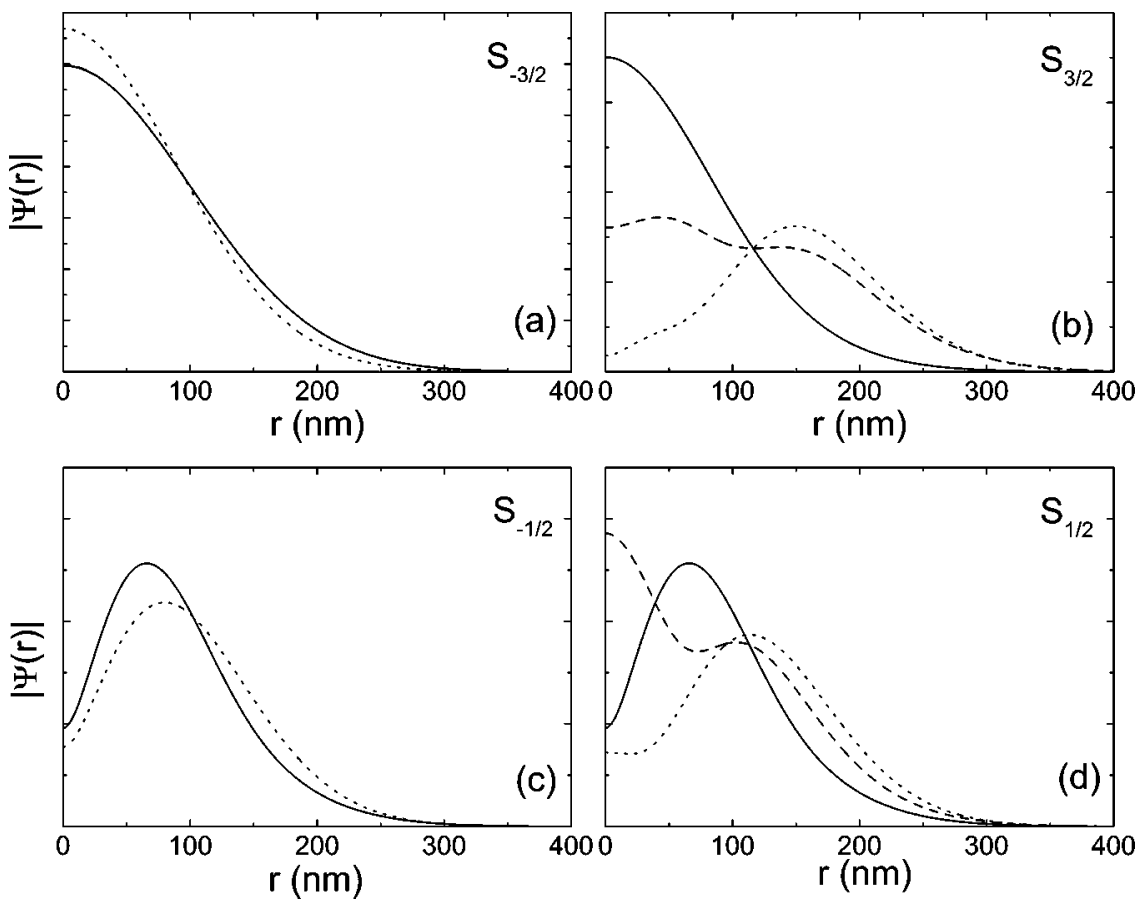

FIG. 5. The wavefunction of the hole ground states for $B=0 \mathrm{~T}$ (the solid curves) and $10 \mathrm{~T}$ (the dotted curves). The dashed curves correspond to the wave function of the hole ground states at $B$ $=1 \mathrm{~T}$ for $S_{-3 / 2}$ and $S_{-1 / 2}$, but $B$ $=0.5 \mathrm{~T}$ for $S_{1 / 2}$, and $1.2 \mathrm{~T}$ for $S_{3 / 2}$. The QD structure is the same as in Fig. 1. 

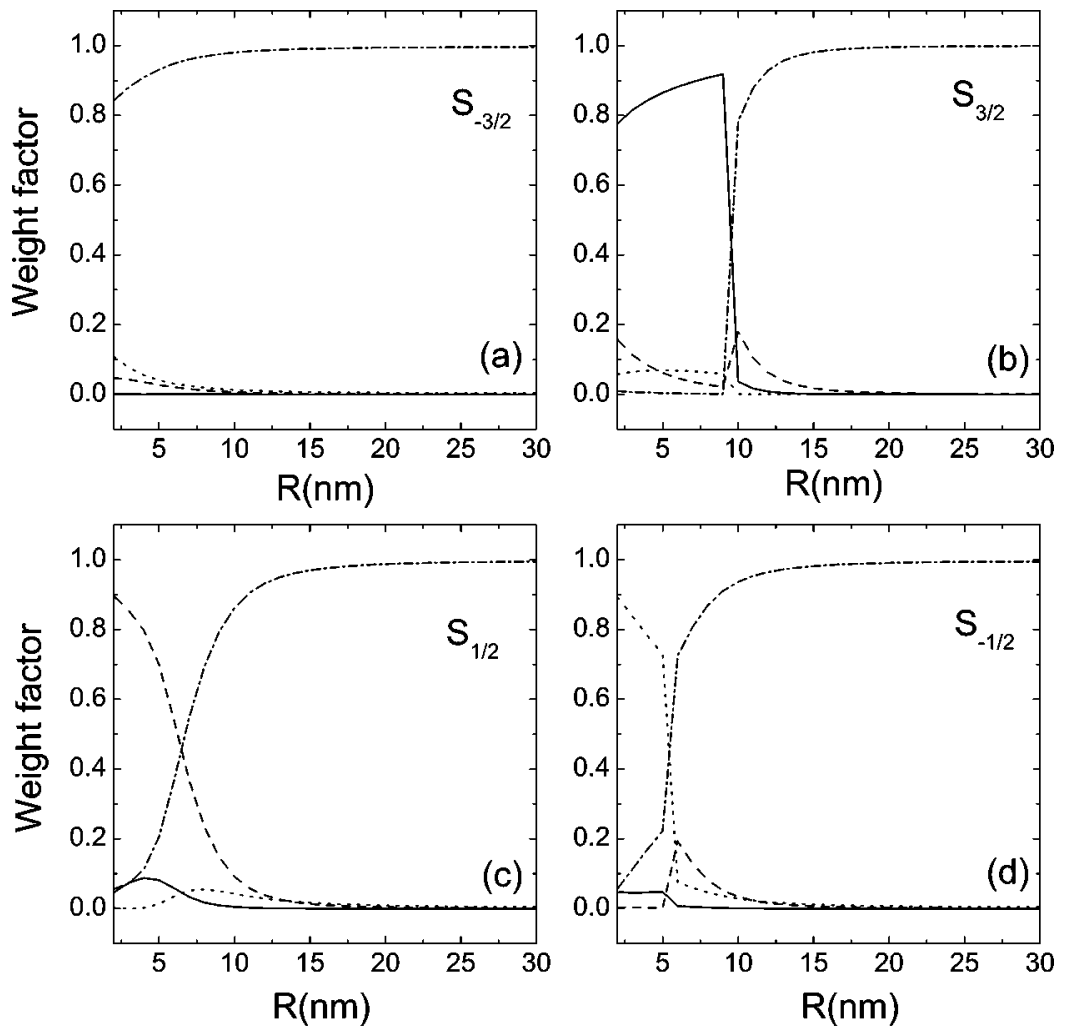

FIG. 7. The weight factor (a)-(d) and the average value (e) of the angular momentum $\left\langle J_{z}\right\rangle$ of the hole ground state for different angular momentum $f_{z}$ versus the QD radius. The QD structure is the same as in Fig. 5.

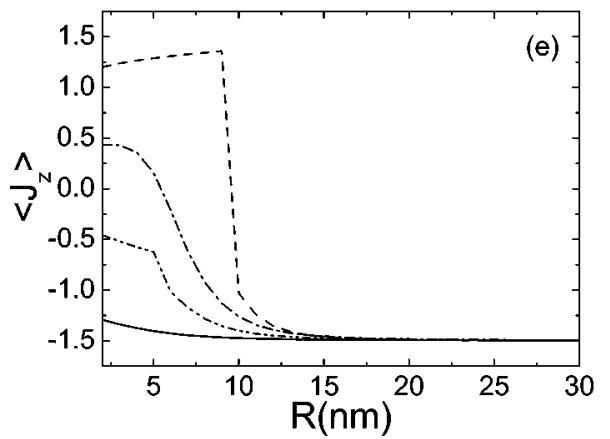

state $S_{-3 / 2}$ also localizes at the center [see Fig. 5(a)], therefore the overlap factor for the transition $S_{-1 / 2} \rightarrow S_{-3 / 2}$ varies slightly with increasing magnetic field. But the wave function of the hole states $S_{3 / 2}$ and $S_{1 / 2}$ change significantly for different magnetic fields. For $S_{3 / 2}$ ground state at zero and small magnetic field, the main component $l=0$ of the wave function is the angular momentum of the envelope function, so it is located at the center of the DMS QD. At large magnetic field $B=10 \mathrm{~T}$, the main component $l=3$ of the wave function of $S_{3 / 2}$ is the angular momentum of the envelope function, the wave function becomes ring-like shape as shown in Fig. 5(b). A similar situation can also be found for $S_{1 / 2}$. From this figure we observe that the wave functions of $S_{3 / 2}$ and $S_{1 / 2}$ change rapidly at the crossing point due to the band mixing effect.

Figure 6 shows how the energies of the electron and hole ground states vary with the QD radius, i.e., the in-plane confinement $\sqrt{\left\langle 0\left|\rho^{2}\right| 0\right\rangle}$, for hole states with different angular momentum $\left\langle f_{z}\right\rangle$. Notice how the energies of the electron and hole decrease as the QD radius increases. The energy spectrum exhibits an interesting anticrossing behavior due to the contribution of the off-diagonal terms in the Luttinger Hamiltonian [see Eq. (2)]. Notice that the ground state of the hole in the DMS QD is changed from $S_{-3 / 2}$ to $S_{-1 / 2}$ when the QD radius decreases, and the first excited state becomes $S_{1 / 2}$ (see the inset). This transition is driven by the quantum confinement effect. The heavy-hole state along the $z$ axis, i.e., the strong confinement direction, has a light-hole character in the $x-y$ plane [see the diagonal terms in Eq. (2)]. With decreasing the QD radius $\sqrt{\left\langle 0\left|\rho^{2}\right| 0\right\rangle}$, the energy of the heavyhole state increases faster than that of the light-hole due to the higher in-plane kinetic energy [the first term of $H_{h h}$ in Eq. (2)], and consequently results in the change of the ground state of the hole in the DMS QD.

Figures 7(a)-7(d) show the weight factors (relative contribution) and the expected value of the angular momentum $\left\langle J_{z}\right\rangle$ in the DMS QD as a function of the QD radius. For the $S_{-3 / 2}$ state, the dominant heavy-hole components is always $|3 / 2,-3 / 2\rangle$, but for the $S_{3 / 2}$ state, the dominant component of the heavy-hole state $S_{3 / 2}$ changes from the components $|3 / 2,3 / 2\rangle$ to the component $|3 / 2,-3 / 2\rangle$ with increasing QD radius. The main components in the $S_{1 / 2}$ and $S_{-1 / 2}$ states are, 


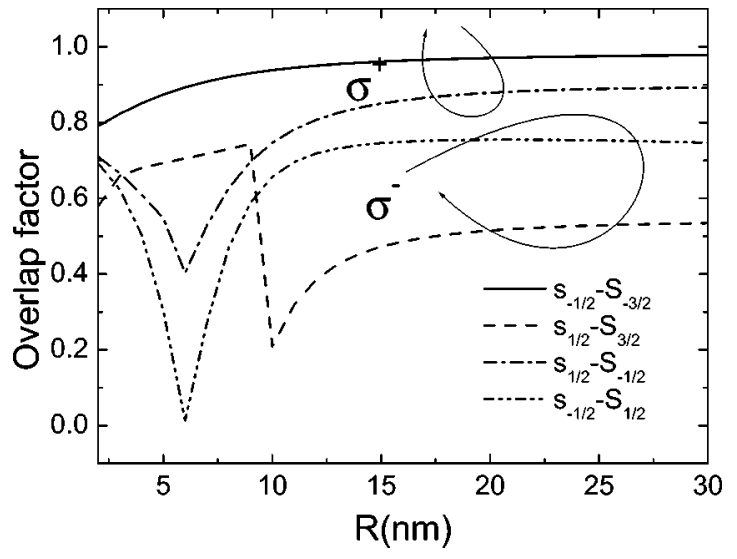

FIG. 8. The overlap factor of the electron and the hole in the DMS QD versus the QD radius. The QD structure is the same as in Fig. 5.

respectively, the component $|3 / 2,1 / 2\rangle$ and $|3 / 2,-3 / 2\rangle$, and the latter becomes dominant for larger QD radius. The average value of the angular momentum $\left\langle J_{z}\right\rangle$ is plotted in Fig. 7(e) as a function of QD radius. For these four hole eigenstates, $\left\langle J_{z}\right\rangle$ decreases with increasing QD radius and approaches a constant $-3 / 2$ for large QD radius. Since the dominant component of the hole eigenstates at large QD radius is the spin-down heavy hole $|3 / 2,-3 / 2\rangle$, therefore $\left\langle J_{z}\right\rangle$ of these four hole eigenstates is equal to $-3 / 2$. Note that $\left\langle J_{z}\right\rangle$ of the hole eigenstates $|3 / 2,3 / 2\rangle$ experience a sharp change from $3 / 2$ to $-3 / 2$ with increasing magnetic field due to the crossover of the hole components $|3 / 2,3 / 2\rangle$ and $\mid 3 / 2$, $-3 / 2\rangle$.

In Fig. 8 we plot the overlap factor $\left|\left\langle\psi_{\sigma}^{e} \mid \psi_{f z}^{h}\right\rangle\right|$ as a function of QD radius. The transitions between the different states display very different features with increasing QD radius, especially at small radius. The transition strength between $s_{-1 / 2}$ and $S_{-3 / 2}$ increases slightly at small QD radius and saturates at large QD radius, but the strength of the transitions $s_{-1 / 2} \rightarrow S_{1 / 2}, s_{1 / 2} \rightarrow S_{3 / 2}$, and $s_{1 / 2} \rightarrow S_{-1 / 2}$ exhibit a dip with increasing QD radius. These features can be understood from the variation of the wave functions of the electron and the hole states when the QD radius changes. The wave functions of hole eigenstates are shown in Figs. 9(a)-9(d) for different QD radius. The wave functions of the $S_{-3 / 2}$ state changes slightly for different QD radius, but the wave functions of the $S_{3 / 2}, S_{-1 / 2}$, and $S_{1 / 2}$ states varies significantly with increasing QD radius due to the change of the dominant component. The main difference can be found in the interband transition strengths for different circular polarization as a function of QD radius arises from the wave functions of the hole eigenstates (see Fig. 7).

\section{CONCLUSIONS}

In summary, we investigated the electronic structure of the DMS QD under a perpendicular magnetic field. Our theoretical calculation shows that the hole energy decreases rapidly for weak magnetic field, and saturates for high magnetic fields, slightly increases again for very strong magnetic field due to the intrinsic Zeeman effect and the magnetic fieldinduced confinement. The ground state of the hole in the DMS QD changes from $S_{-3 / 2}$ to $S_{-1 / 2}$ with decreasing QD radius. It is interesting to note that the mixing of different hole components in the hole states can be tuned significantly by changing the external magnetic field and the in-plane confinement, and consequently influences the strength of the interband transitions. The external magnetic field provides us with a powerful tool to tailor the electronic structure of a DMS QD via the $s p-d$ exchange interaction. Here we concentrate on the magnetic field dependence of the single electron and hole states. The Coulomb interaction between electron and hole was neglected in our calculation which is important for the optical property of DMS QD, ${ }^{5}$ especially in
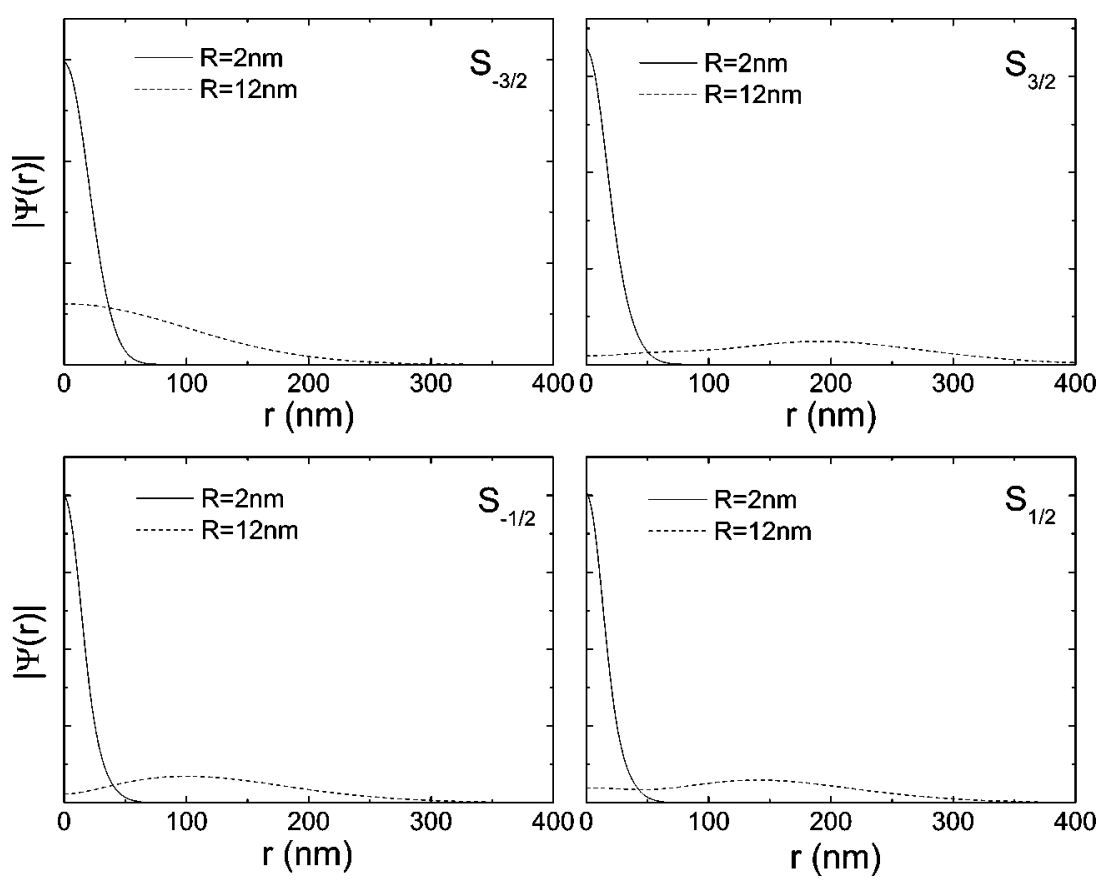

FIG. 9. The wave function of the hole ground states for different $S_{f_{Z}}$ for two values of QD radius $R=2 \mathrm{~nm}$ and $12 \mathrm{~nm}$. 
the case of weak confinement. The Coulomb interaction can lower the transition energy and enhance the transition strength. Nevertheless, our numerical results about the transition energy and strength are qualitatively correct especially in the strong confinement regime. However, the electronic structure and the optical property of the DMS QD under an external magnetic field are expected to play an important role in the physics of spintronic nanodevice.

\section{ACKNOWLEDGMENTS}

This work was partly supported by the NSFC No. 60376016 and No. 90301007, the special fund for Major State Basic Research Project No. G001CB3095 of China, the Flemish Science Foundation (FWO-Vl), Belgian Science Policy and the Bilateral Cooperation programme between Flanders and China.
*Electronic mail: kchang@red.semi.ac.cn

${ }^{1}$ B. E. Kane, Nature (London) 393, 133 (1998).

${ }^{2}$ D. Loss and D. P. DiVincenzo, Phys. Rev. A 57, 120 (1998).

${ }^{3}$ J. K. Furdyna, J. Appl. Phys. 64, R29 (1988).

${ }^{4}$ P. A. Wolff, in Semiconductors and Semimetals, Diluted Magnetic Semiconductors, edited by J. K. Furdyna and J. Kossut (Academic, New York, 1988).

${ }^{5}$ K. Chang, J. B. Xia, and F. M. Peeters, Appl. Phys. Lett. 82, 2661 (2003).

${ }^{6}$ S. H. Xin, P. D. Wang, A. Yin, C. Kim, M. Dobrowolska, J. L. Merz, and J. K. Furdyna, Appl. Phys. Lett. 69, 2884 (1996); Y. Oka, J. Shen, K. Takabayashi, N. Takahashi, H. Mitsu, I. Souma, and R. Pittini, J. Lumin. 83, 83 (1999); C. S. Kim, M. Kim, S. Lee, J. Kossut, J. K. Furdyna, and M. Dobrowolska, J. Cryst. Growth 214, 395 (2000).

${ }^{7}$ A. A. Maksimov, G. Bacher, A. MacDonald, V. D. Kulakovskii, A. Forchel, C. R. Becker, G. Landwehr, and L. W. Molenkamp,
Phys. Rev. B 62, R7767 (2001); G. Bacher, H. Schomig, M. K. Welsch, S. Zaitsev, V. D. Kulakovskii, A. Forchel, S. Lee, M. Dobrowolska, J. K. Furdyna, B. Konig, and W. Ossau, Appl. Phys. Lett. 79, 524 (2001).

${ }^{8}$ J. Kossut, I. Yamakawa, A. Nakamura, I. Yamakawa, A. Nakamura, and S. Takeyama, Appl. Phys. Lett. 79, 1789 (2001).

${ }^{9}$ J. M. Luttinger, Phys. Rev. 102, 1030 (1956).

${ }^{10}$ R. C. Ashoori, H. L. Stormer, J. S. Weiner, L. N. Pfeiffer, S. J. Pearton, K. W. Baldwin, and K. W. West, Phys. Rev. Lett. 68, 3088 (1992); Ch. Sikorski and U. Merkt, ibid. 62, 2164 (1989); A. Kumar, S. E. Laux, and F. Stern, Phys. Rev. B 42, 5166 (1990); P. Hawrylak, Phys. Rev. Lett. 71, 3347 (1993).

${ }^{11}$ F. B. Pederson and Y. C. Chang, Phys. Rev. B 55, 4580 (1997).

${ }^{12}$ J. Siviniant, F. V. Kyrychenko, Y. G. Semenov, D. Coquillat, D. Scalbert, and J. P. Lascaray, Phys. Rev. B 59, 10276 (1999).

${ }^{13}$ A. K. Bhattachajee, Phys. Rev. B 55, 10613 (1997). 